\title{
Le hate dans les commentaires des internautes de la presse sportive
}

\author{
Internet users' hate phenomenon in the sports press
}

\author{
Andrzej Napieralski \\ Université de Łódź \\ napieralski.a@op.pl
}

\begin{abstract}
In recent years, the Internet has become a place where a new type of users, the "outraged", have the opportunity to express themselves anonymously on any subject. They take advantage of it to propagate hate just for pleasure. This kind of verbal abuse, which we will consider as a new act of speech, already occupies an important place in the community of web users. This research will examine the hate speech of French football fans, in order to analyze their ways of expressing themselves. It seems that verbal hate is characteristic of younger people, the generation not shocked by the deviant "trolling" behaviors. Hate is expressed in most cases with insults, but it seems to us that it is not the lexemes and their semantic content that play the preponderant role, but above all the context and co-text of the utterances produced by Internet users. Arriving at the definition of the hate phenomenon will be one of the main objectives of this study. We will try to prove that this concept is not identical to those already well-defined, like insults, curses, valves, etc. The hate phenomenon will be illustrated with several examples taken from a corpus of French and Polish comments.
\end{abstract}

Keywords: football, hate, sociolinguistics, discourse analysis, insult, internet commentary.

\section{INTRODUCTION}

L'apparition de ce moyen de communication qui est internet a bouleversé le monde de la communication. La création d'un espace qui permet à toutes les personnes de s'exprimer librement sur divers sujets a donné naissance à un nouveau type d'échanges entre locuteurs. L'existence des sites du type: forums internet, blogs, journaux en ligne, réseaux sociaux et l'accès global à la toile ont provoqué 
une révolution dans la communication entre les humains. Certes, l'outil en tant que tel (internet) est une idée qui a priori avait l'intention d'améliorer les relations entre les gens, de supprimer l'espace et le temps entre l'énonciation et la réception d'un message pour permettre à une communication instantanée. Toutefois, l'évolution de l'internet (mis à part un accès plus rapide à toutes sortes d'informations) a provoqué des effets secondaires. La possibilité de partager ses idées, d'écrire librement ce que l'on pense d'une façon pour que chacun puisse en prendre conscience a eu des effets néfastes qui étaient à prévoir tenant compte de la nature humaine.

L'objectif du présent travail sera de montrer que la liberté d'expression donne naissance au partage de la société et, par conséquent, à la marginalisation en groupes qui chercheront à marquer leur identité en s'excluant et en excluant les autres. Le fait que les gens soient différents permet de débattre, de confronter les idées, d'apprendre à respecter d'autres points de vue, d'autres cultures et d'élargir la conceptualisation du monde. Cependant, notre nature humaine nous pousse parfois à nous obstiner sur nos convictions en rejetant par défaut les opinions des autres. Le dialogue et l'art de la communication sont très souvent remplacés par un figement d'idées et une attitude d'obstruction envers un différent point de vue. Nombreux sont ceux qui sont sourds au dialogue et dont le seul but est de manifester une appartenance à un groupe en proclamant des idées et des opinions figées tout en déniant sans réfléchir les idées de l'opposition. Il est vrai que toute personne a le droit de s'exprimer librement, mais il n'est pas moins vrai que la communication devrait se tenir dans l'esprit d'un respect mutuel pour les idées. L'existence des extrémistes de la pensée et de l'intolérance entre les gens a fait naître le concept du hate speech ('discours de la haine') qui est considéré comme un acte de parole inapproprié et bouleversant les limites du bon sens. Ce type de discours est puni par la loi, car il relève des sentiments humains les plus sombres et de la mauvaise foi. Le Conseil de l'Europe a défini ce concept dans une recommandation du 30 octobre 1997 où on peut lire que :

The term "hate speech" shall be understood as covering all forms of expression which spread, incite, promote or justify racial hatred, xenophobia, anti-Semitism or other forms of hatred based on intolerance, including: intolerance expressed by aggressive nationalism and ethnocentrism, discrimination and hostility against minorities, migrants and people of immigrant origin ${ }^{1}$.

Cette recommandation qui précise ce qui n'est pas admis permet de combattre les extrémistes, cependant cela ne permet pas de résoudre le problème de l'animosité

\footnotetext{
${ }^{1}$ fr. « Le terme hate speech devrait être compris comme englobant toutes formes d'expressions qui propagent, incitent, promeuvent ou justifient la haine raciale, la xénophobie, l'anti-sémitisme ou autres formes de haine basées sur l'intolérance y inclus : l'intolérance qui s'exprime dans un nationalisme ou un ethnocentrisme agressif, une discrimination ou hostilité envers des minorités, des migrants et des gens d'origine étrangère ». [traduction de l'auteur]
} 
entre les gens et le désir de nuire à autrui qui est le propre de certaines personnes. Internet a permis le développement d'autres formes de manifestation des idées et du désir de nuire. Le trolling est un concept plutôt récent qui est le fruit de l'apparition de la liberté d'expression sur internet. De manière générale, ce concept exprime l'attitude consistant à provoquer les participants à une discussion en ligne, par exemple en lançant systématiquement des débats sur des sujets très polémiques ou en agressant les participants au débat, avec un but avant tout de faire dégénérer la discussion. Le trolling est surtout le domaine des gens jeunes qui l'utilisent pour manifester leur mal de vivre, toutefois, il peut se développer en outil de propagande servant à manipuler les lecteurs des informations sur la toile. Les dernières campagnes électorales ${ }^{2}$ ont démontré que l'activité des trolls $^{3}$ est une arme de propagande inestimable. Les trolls peuvent être divisés en deux types, ceux qui aiment quand la communication dégénère et qui en tirent un vicieux plaisir et ceux qui sont payés afin de mener une campagne de propagande, par exemple sur les réseaux sociaux. Le trolling est une pratique virale qui se répand très vite du fait que certains utilisateurs d'internet se laissent manipuler par des propos qui sont souvent mensongers, mais qui répondent à leurs convictions politiques. De tels propos sont ensuite partagés sur les réseaux sociaux et ce sont les utilisateurs eux-mêmes qui font circuler ces informations sans prendre l'effort de vérifier leur véracité ${ }^{4}$.

Manifester la haine (hate speech) et saboter une communication (trolling) ont donné naissance à une attitude des internautes qui faute de mieux sera défini comme hate dans le présent travail. Ce que nous comprenons par le concept hate est l'emprunt du concept polonais 'hejt' ${ }^{5}$ qui englobe un type de comportement proche du trolling (sans la volonté de faire dégénérer la discussion) qui diffère du hate speech par son aspect surtout persifleur et dépourvu de discrimination raciale ou ethnique. La personne qui s'adonne au hate est un hejter (hater) ('rageux') ${ }^{6}$. Le concept évolue de plus en plus du commentaire malicieux vers la moquerie. Le hate est une extension du sens du signifié du mot anglais tout comme sa dérivation le substantif hater 'rageux' (la personne qui s'adonne au hate). On retrouve une de ses premières traces dans la chanson du rappeur américain Dr. Dre - Still D.R.E de l'album « 2001 » sorti en 1999 où il chante : Ladies, they pay homage, but haters

\footnotetext{
${ }^{2}$ Par ex. la campagne électorale aux élections gouvernementales en Pologne (2015) ou la campagne électorale présidentielle aux États-Unis (2016).

${ }^{3}$ L'existence et l'activité des trolls sur internet ne peut être prouvée, les personnes qui s'occupent de se type de pratiques ne s'en ventent pas. Parfois il est difficile de distinguer l'activité d'un troll de l'activité d'un utilisateur quelconque de la toile, cependant il est courant que certaines personnes profèrent des propos controverses non pas par convictions mais par appât du gain.

${ }^{4}$ Cette pratique est connue sous le nom de fake news (fausses informations).

5 'hejt' est une assimilation graphique du mot anglais hate.

${ }^{6}$ Extension de sens du mot créé par concentration de l'expression « avoir la rage » qu'on retrouve par ex. dans (Ferrier, 2003, p. 310).
} 
say Dre fell off... ${ }^{7}$. Le hate semble devenir une pratique des internautes qui gagne de plus en plus en popularité, elle est partagée par un grand nombre d'utilisateurs de la toile et il semble même qu'elle soit le signe identitaire d'un certain groupe de gens. Dans son livre Le parler ordinaire William Labov présente le concept de la vanne qui est une remarque ou allusion désobligeante à l'adresse de quelqu'un. Labov constate que cela est un rituel à fonction identitaire : «c'est que la façon dont les vannes s'échangent et sont évaluées par le groupe suit un modèle rituel établi qui reflète des conventions et un savoir social en majeure partie étrangers aux membres des autres sous cultures "(Labov, 1993, p. 401). Au cas du hate, il pourrait s'appliquer la même observation, le caractère persifleur et moqueur des propos des haters doit être évalué et accepté par d'autres internautes qui réagiront à ces propos. Le manque de réaction quelconque fait tout de suite couler le commentaire et met l'énonciateur dans une position de manque d'acceptation. Il faut souligner que la réaction ne doit pas être forcement favorable, une réponse ou riposte négative met aussi en valeur le hate propagé. Les rageux (haters) constituent une communauté d'internautes qui peuvent être reconnus grâce aux types de comportements adoptés et à la façon d'exprimer leurs paroles. Pour montrer un certain éventail de type de rageux nous allons présenter un classement plutôt ludique et arbitraire du site www.nioutaik.fr ${ }^{8}$ qui permettra de voir les différents comportements qui entrent dans le cadre des variantes du hate.

- Un gros bœuf: il insulte, il écrit avec le caps lock appuyé, il utilise beaucoup de signes diacritiques.

- Un Joe-le-péteux : insulte d'une façon plus subtile en utilisant un français standard/soutenu (l'utilisation de l'euphémisme).

- Un Fufu : (de furtif) il pose des questions contestataires, il utilise le „oui mais”.

- Ranafout: (de rien à foutre) essaye à tout prix de trouver sa place dans la discussion, même quand il n'est pas concerné.

- Max la menace : il profère des menaces envers les autres participants.

Ce classement permet de se rendre compte que le discours que tiennent les rageux et par conséquent le hate en tant que tel sont très diversifiés tant au niveau du choix du lexique qu'au niveau de la forme que détient le message.

\section{PRÉSENTATION DU CORPUS}

L'objectif du présent travail est d'illustrer le hate à l'aide de certains exemples afin de pouvoir remarquer des traits particuliers de ce discours. Dans ce but, nous

\footnotetext{
${ }^{7}$ Les femmes, elles rendent hommage, mais les haters disent que Dre est tombé. [traduction de l'auteur]

${ }^{8} \mathrm{http} / / /$ www.nioutaik.fr/index.php/2013/04/09/645-comment-faire-un-bon-commentaire- de-rageux
} 
nous proposons de présenter un corpus composé de commentaires d'internautes qui provient de la presse sportive et plus précisément du site www.lequipe.fr. Les commentaires concernent les articles liés au football. Les titres des articles dans lesquels le corpus a été trouvé sont :

I. Blanc a perdu gros (du 14/04/2016) ${ }^{9}$

II. La fondation de Didier Drogba accusée de fraude (du 14/04/2016) ${ }^{10}$

III. La vente de l'OM, " une bonne nouvelle » selon Luc Laboz, le directeur général du club (du 14/04/2016) ${ }^{11}$

IV. Karim Benzema : Raymond Domenech aurait pris la même décision que la FFF (du 14/04/2016) $)^{12}$

V. Bleus : Karim Benzema ne sera pas sélectionné pour l'Euro (du 13/04/2016) ${ }^{13}$

VI. Atlético-Barça : la main de Gabi était-elle dans la surface? (du 13/04/2016)

Le choix des articles n'est pas tout à fait arbitraire, les commentaires apparaissent le plus souvent sous des articles à controverse comme les matchs tendus entre les équipes réputées pour animosités entre leurs supporters ou les articles parlant de personnages du milieu qui ne sont pas neutres à l'égard des fans du football. Les émotions jouent un rôle important chez les rageux qui ne s'intéressent pas aux informations du monde du football, mais à la polémique qu'ils peuvent créer en publiant leurs points de vue. Les commentaires sont de différente nature, on retrouve ceux qui se réfèrent directement au contenu de l'article et ceux qui sont des réactions aux réponses des autres internautes. La construction des commentaires varie au niveau de la syntaxe, cela peuvent être de simples locutions, des syntagmes, des lexèmes ou des phrases interrogatives comme :

(1) greg_44. C'est qui lui? On s'en bat les ... ${ }^{15}$.

(2) arctarus. Qu'en pense Aimé Jacquet? Et même Michel Hidalgo ? et ma concierge?

L'expressivité du message publié peut être soulignée à l'aide d'une phrase exclamative :

\footnotetext{
${ }^{9}$ https://www.lequipe.fr/Football/Article/Laurent-blanc-a-perdu-gros/652272

${ }^{10} \mathrm{https}: / /$ www.lequipe.fr/Football/Actualites/La-fondation-de-didier-drogba-accusee-de-fraude/652312

${ }^{11} \mathrm{https}: / /$ www.lequipe.fr/Football/Actualites/La-vente-de-1-om-une-bonne-nouvelle-selon-luc-labozle-directeur-general-du-club/652304

${ }^{12} \mathrm{https}$ ://www.lequipe.fr/Football/Actualites/Karim-benzema-raymond-domenech-aurait-pris-la-me me-decision-que-la-fff/ 652339

${ }^{13} \mathrm{https}: / /$ www.lequipe.fr/Football/Actualites/Bleus-karim-benzema-ne-sera-pas-selectionne-pour-1euro/652142

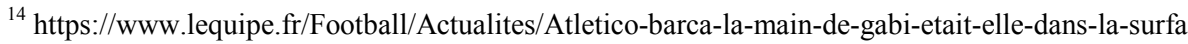
ce-video/652278

${ }^{15}$ Les exemples tirés du corpus seront illustrés selon le modèle : le numéro du commentaire (1), le nick 'surnom' de l'internaute qui l'a posté 'écrit' et le commentaire en italique. Tant la graphie des surnoms que la forme exacte du commentaire lui-même seront gardés pour respecter l'authenticité des formes utilisées par les internautes.
} 
(3) Orvis. "Ahhh, vous voyez bien que je sais prendre les bonnes décisions! ». Au secours.

(4) LeParisienDu12. Quelle pipelette, la Raymonde!

La forme d'un commentaire est souvent une stylisation d'un message particulier comme un slogan, une publicité, ou une imitation d'une annonce comme l'exemple qui suit :

(5) vivemezigue. Recherche repreneur inconscient capable de mettre beaucoup d'argent, sans retour sur investissement, pour remettre en état un club en ruines non entretenu, avec des supporters insupportables des joueurs sans volonté. Prévoir dirigeants compétents et directeur sportif connaissant le football ainsi qu'un entraineur avec le même profil. Faire offre.

Les propos tenus dans les commentaires sont des choix conscients des internautes qui les publient. Le message qui est divulgué contient aussi bien un sens précis que le locuteur souhaite transmettre qui est le plus souvent habillé d'une forme qui n'est pas forcement neutre. Les internautes utilisent des figures d'une manière plus ou moins consciente. L'analyse des figures qui se trouvent dans les énonciations des participants de la discussion sera effectuée selon le classement d'Olivier Reboul en figures de pensée, figures de sens et figures de mots (Reboul, 2011).

\section{ANALYSE}

Les figures de pensée qui concernent les rapports entre les idées dans le discours entier sont le plus souvent représentées par l'ironie. Cette figure qui permet d'exprimer à l'aide des mots le contraire de ce qu'on veut faire entendre peut être explicite ou implicite. Nous comprenons par ironie explicite un énoncé qui manifeste l'écart entre le sens des lettres et celui de l'esprit qui ne demande pas une connaissance plus approfondie du sujet qui provoque la polémique. Ainsi, dans l'exemple (6) nul n'a besoin ni de connaissance du contenu de l'article ni d'un savoir approfondi du domaine en question :

(6) phil15. t'as pas les numéros sortants du prochain loto, stp?

Cela n'est pas le cas du prochain exemple qui reste toutefois explicite du fait que son sens appartient au savoir général de tous les adhérents du forum de discussion de l'Équipe. Dans (7) l'ironie repose sur le fait que les joueurs à six mois de la fin de leur contrat peuvent changer de club librement et les clubs ne peuvent pas tirer de profits de leur transfert.

(7) JMHoleAss. tu vends des joueurs en fin de contrat? je te prends comme conseiller patrimonial si t'es libre. 
Réussir à vendre un joueur en fin de contrat serait comme réussir à vendre à quelqu'un un produit qu'il pourrait normalement recevoir gratuitement. Le fait de faire une remarque ironique polémique avec le contenu de l'article reste explicite, dans (8) l'auteur du commentaire manifeste son indignation envers l'attitude du joueur qui a créé une fondation de charité qui s'est penchée vers la fraude (article IV) :

(8) vivemezigue. J'ai décidé de créer un fondation en ma faveur avec un but lucratif, je vous remercie de votre générosité.

Une ironie peut être accompagnée d'une hyperbole pour renforcer l'effet moqueur; c'est le cas de :

(9) MyKeulKeul69. Le tout est de retrouver un repreneur sérieux. De toute façon, la situation actuelle de l'OM ne doit pas durer. Parce que là, on rigole, mais depuis la dernière victoire Marseillaise au Vélodrome, on a changé 2 fois d'heure :-).

Dans ce commentaire de l'article III, l'intension de l'émetteur du message est de persifler le club phocéen en difficulté sportive et, par conséquent, inciter les supporters de ce club à la réaction. Ce que nous comprenons par l'ironie implicite, c'est quand la connaissance du contenu de l'article ne peut être suffisante pour comprendre la portée de cette figure dans une énonciation. Ainsi, certaines réponses à l'article III contiennent un sens qui peut être compris uniquement par les personnes qui s'intéressent au sport, à l'actualité sportive et au monde autour du sport. Dans l'exemple (10), on a affaire à l'annonce du fameux joueur de basket qu'il se retire du sport professionnel, l'internaute a utilisé cette information afin de faire une remarque insolite que le sportif aisé pourrait racheter le club en mauvaise situation financière.

(10) Yeshol. Kobe va racheter l'OM.

Une autre réaction persifleuse c'est (11) qui prétend que le chanteur en difficulté financière récemment annoncée pourrait se permettre de racheter le club.

(11) abas64. Gilbert montagné est sur le coup.

Dans l'exemple (12), pour comprendre l'ironie il est indispensable de connaître le personnage de Stephane Plaza qui anime à la télévision l'émission "Chasseur d'appart' » et qui pourrait selon le modèle du concept « Home Staging ${ }^{16}$ » vendre le club marseillais.

(12) Dearduke. Il ne reste plus qu'à faire venir Stéphane Plaza pour faire du club staging, et trouver un acheteur:)

\footnotetext{
${ }^{16}$ Concept qui tente de valoriser, à peu de frais, un bien immobilier dans le but de le vendre à meilleur prix et dans les plus brefs délais.
} 
Le nombre important de commentaires relevant du hate qui se trouvent sous l'article III résulte du fait que la nouvelle concernant la vente du club circule depuis plusieurs mois dans la presse. Le nombre de différents investisseurs potentiels que les médias ont déjà mentionné dans ce contexte fait que les rageux qui ont une bonne mémoire ne manquent pas de rappeler des noms au moment approprié. Tel est le cas de Jack Kachkar, un homme d'affaires canadien qui fut à maintes reprises mentionné par la presse dans le contexte de l'achat de l'Olympique de Marseille :

(13) StellaRossa. Que la fête commence! C'est la tournée des charlots aux pays des sardines. 1, 2, 3 dis Kachkar!

Dans les répliques que font les lecteurs de lequipe.fr, il est possible de trouver d'autres types de figures de pensée. On a repéré des exemples de prolepse qui est une figure dont le but est de retourner l'argument contre l'auteur. Dans l'article II il est question d'hôpitaux vides du fait qu'ils ne fonctionnent pas à cause du manque de fonds. Cela a permis à un hater de retourner cet argument (14) afin de voir le bon côté des choses.

(14) Giman. Un hôpital vide, c' est plutôt une bonne nouvelle ...

Une autre figure de pensée repérée dans le corpus des commentaires c'est la contrefision qui est une suggestion du contraire de ce qui est dit. Dans la phrase (15) l'affirmation nos chers amis Marseillais est loin d'être une déclaration de sympathie envers ce club.

(15) Le coq Gaulois. En espérant un repreneur aussi propre que les Qataris à Paris, juste pour voir comme ça les réactions de nos chers amis Marseillais ...

Dans l'exemple (16) nous avons affaire à un type d'apodioxie donc d'un refus argumenté d'argumenter qui a comme fonction de négliger les propos de l'article (ici IV) :

(16) cariocabrazilian. Qui s'en fout ????

Un autre type de figures qu'Olivier Reboul mentionne dans son livre sont les figures de sens. Parmi celles-ci, ce sont surtout les métaphores et les comparaisons qui peuvent être trouvées dans les commentaires des internautes. Étant donné que l'objectif principal des rageux est de stimuler les autres participants de l'échange en ligne par la profession de propos moqueurs et persifleurs, cela n'étonne pas qu'on trouve des commentaires négatifs envers des protagonistes du football à qui on ne prête pas beaucoup d'estime. La comparaison est surtout de nature négative, dans le cas (17), l'ancien entraîneur de l'Équipe de France de football est comparé à un tacle, par derrière donc une des fautes les plus graves qu'un joueur peut se permettre de faire sur la pelouse : 
(17) Padawan44. Tu le sors par la porte, il revient par la fenetre, tactiquement trop fort Raymond, comme tes tacles par derrière.

On retrouve aussi l'exemple (18) où Raymond Domenech est considéré comme un virus du fait de sa présence trop importante dans les médias.

(18) lamolie. ce mec est pire qu'un virus qu'il se taise à jamais.

Il faut signaler que les personnes atteintes par le hate sont rarement des victimes innocentes, certes il n'est guère justifié d'approuver ce type de comportement, toutefois il faut remarquer que les personnes visées sont des protagonistes qui se sont inscrits dans l'esprit collectif du monde du football par des actes qu'ils ont commis. Raymond Domenech est considéré par la plupart des Français comme responsable de l'humiliation qu'a subi l'Équipe de France de football pendant la Coupe du monde de 2010 en Afrique du Sud, les Français ne sont pas sortis de la phase de poule. Dans le commentaire (19) ce sont Pape Diouf et José Anigo, les directeurs du club phocéen qui ne sont pas très appréciés par les supporters marseillais qui sont l'objet de la métaphore avilissante.

(19) pascaloudu13. les vautours à l'affût se positionnent (DIOUF ANIGO).

Les figures de mots que nous retrouvons dans les exemples repérés sont surtout des figures qui jouent sur les sons des phonèmes, des syllabes ou des mots. L'antanaclase qui joue sur les sens différents d'un même mot peut être illustrée par l'exemple (20) où il y a le jeu sur le mot « Ivoirien » dans lequel on vise à confondre le nom de l'habitant de la Côte d'Ivoire avec le sens qui découle d'une autre interprétation de son signifiant « Il ne voit rien ».

(20) THE_CRITICAL_ONE. Un Ivoirien accusé de Fraude, il va encore nous dire qu'il n'a rien $v u$.

La dérivation comme association d'un mot à un autre de même racine est une pratique ludique souvent utilisée par les internautes. Dans l'exemple (21) l'internaute ne manque pas de défiger son intention qui consistait à rapprocher les lexèmes Qatari (habitant du Qatar) avec une déformation contenant le redoublement hypocoristique du langage enfantin « caca ».

(21) philou-21. blanc n 'a rien perdu puisque blanc n 'a rien...gagné. et ce psg kakari ne gagnera JAMAIS rien (kakari pour qatari).

Un autre exemple de ce type c'est l'énonciation (22) dans laquelle le patronyme du footballeur Karime Benzema est devenu une fracto-composition (selon le tableau des matrices internes instauré par Jean-François Sablayrolles; voir Sablayrolles, 2015 et $c f$. 2017) liant le nom du joueur avec le mot «bête » pour déprécier celui-ci. 
(22) gil06. Super bêtezema aura du temps pour apprendre la marseillaise.

Dans l'exemple qui suit la substitution du ' $\mathrm{t}$ ' de Catalan par un ' $\mathrm{k}$ ' accompagné d'un changement intentionnel de la graphie 'an' en 'ent' donne naissance à la forme « cakalent » à valeur de double jeu de mot :

(23) fdcap. eh oh les cakalents elle a joué la messi car pas vu.

Dans les commentaires qui expriment le hate, il est possible de trouver des exemples d'argumentations. Dans ce discours que sont les commentaires des internautes, des arguments relevant de l'approche pragma-dialectique sont possibles à être repérés. Ainsi, dans l'exemple (24) le syntagme Les africains sont coutumiers $d u$ fait peut être interprété comme un paralogisme de composition, c'est-à-dire qui repose sur la confusion des propriétés des parties et du tout.

(24) f_philippot. Les africains sont coutumiers du fait, souvenez-vous Boli (tiens, un ancien marseillais aussi) et mouss diouf (pour ne citer qu'eux) et après ça vient donner des leçons aux autres.

L'utilisation d'une constatation générale s'appliquant à tous les Africains n'est pas un argument logiquement valide. Il est de même dans l'exemple qui suit dans lequel on laisse supposer que le joueur de football en question (Didier Drogba) soutient un politicien inculpé de crimes contre l'humanité juste par le fait d'être ivoirien :

(25) waldemar victorino. étonnant, de la part d'un ancien soutien de Gbagbo, vraiment étonnant...

Un autre argument qui a été repéré dans le corpus c'est celui de a contrario qui est un raisonnement dont la forme est identique à celle d'un autre, mais dont l'hypothèse et, par conséquent, la conclusion sont inverses de celui-ci. Dans l'exemple (26), il semble que la suggestion de l'auteur de ces propos soit de mettre en valeur le club Olympique de Marseille tout en dépréciant celui du Paris SaintGermain, mais la vraie intention est de ridiculiser le club marseillais par un sens ironisant découlant de ces propos.

(26) THE_CRITICAL_ONE. Ya bien des pigeons qui ont rachetés le PSG, alors un vrai club avec une vraie histoire et des vrais supporters ça va se vendre facilement.

Le hate qui est le propre de certains internautes, mis à part sa forme qui varie selon le choix de l'utilisateur est surtout une attaque dirigée contre une personne ou un groupe de personnes. Les attaques qui se font sur la toile sont le résultat d'une frustration à l'égard d'un certain statu quo qui touche les émotions de l'énonciateur du message. Les principales attaques qui ont pu être repérées ce sont celles qui touchent 
un protagoniste, un autre internaute ou un club adverse. Dans l'exemple (27) le rageux critique l'entraîneur du club (ici Laurent Blanc) en imputant aux dirigeants que ce club fonctionne sans entraîneur.

(27) adyabeba. Le PSG ne gagnera pas la LDC... sans entraineur...

Le manque de respect envers un protagoniste peut se faire tant au moyen d'une simple insulte ou injure ou par l'utilisation de l'expressivité par les signes typographiques utilisés. Dans l'énoncé qui suit l'internaute frustré critique Zlatan Ibrahimovic en ironisant et mettant le substantif monsieur en majuscules pour montrer ses émotions négatives :

(28) Garcia. En 1995, le PSG était allé en demi-finale contre AC Milan (...) Ils existaient donc plus que le grandd joueur de ligue 1 mais petit joueur de C1 qu'est MONSIEUR Ibrahimovic.

Une attaque peut se faire aussi à la manière d'un sous-entendu. Dans la phrase (29), l'auteur du commentaire laisse supposer que le joueur en question (ici Serge Aurier) aurait un traitement favorable de la part du propriétaire du club.

(29) JMHoleAss. Peut être mais la Prince a publiquement applaudi le retour du cousin.

Parmi les attaques dirigées contre les protagonistes on retrouve aussi des pseudonymes et des sobriquets qui témoignent de la volonté des auteurs des propos d'avilir les footballeurs. Dans l'exemple (30), l'internaute utilise la forme $f c$ pepito pour ridiculiser le joueur André-Pierre Gignac qui joue au Mexique. Le coupeur de citrons est une collocation qui dans le jargon du football se réfère à un joueur qui est souvent remplaçant (ici le cas d'Olivier Giroud du club Arsenal Londres).

(30) groove33. Ouais allee parlons du fc pepito et du coupeur de citrons d'arsenal.

La diffusion d'un article qui soulève une thématique délicate du fait qu'il donne la chance à la polémique aux supporters de deux clubs adverses (tel l'article III), a comme répercussion l'apparition d'un grand nombre de rageux qui attendent ce type d'articles avec impatience. Le conflit entre les supporters qui est le résultat de l'existence d'une culture hooligan n'épargne pas les différents terrains d'échanges verbaux sur la toile. L'espace « réactions » sous les articles de la presse sportive de lequipe.fr en est un. Les internautes ont conscience de l'existence dans le groupe d'une certaine convention et d'un certain modus operandi en ce qui concerne le caractère des propos introduits sous les articles. Le commentaire (31) n'a pas plu à un grand nombre de participants de l'échange qui ont vite constaté que celui-ci n'était pas familier avec les règles tacites qui sont en vigueur sur ce site. 
(31) syl2alf. On reconnait bien les lâches, passant de la flagornerie au lynchage public! Ils sont beaux les corbeaux de l'Equipe! Mais qui voudrait payé pour lire de pareils sornettes, cachez vous derrière le payant, vos étrons n'en seront que moins nocifs...

La réponse d'un autre internaute (32) lui a signalé que sur ce site les intervenants étaient plus jeunes et qu'ils utilisaient un différent style tant dans le choix du lexique que de la syntaxe.

(32) Hubert-ALLESSE. $s y l+50$.

Les attaques entre internautes visent à déprécier l'adversaire en le discréditant au sein du groupe. Si l'exemple (32) critiquait un internaute du fait de son âge trop mûr pour ce type d'activité, il n'est pas moins rare de rencontrer des arguments opposés qui visent à porter atteinte à quelqu'un pour des raisons de manque de maturité ou d'expérience. Dans l'exemple (33), on retrouve les appellations Facebook et twitoos (pour parler des internautes utilisateurs de ces sites internet) qui impliquent un mépris envers ce type de personnes qui ne connaissent le sport que grâce aux réseaux sociaux sur lesquels ils passent leur vie.

(33) nasri_13300. stella l'élu des Charlot c'est bien toi juste pour info l om est toujours le club le plus titré et le plus suivit de France. je parle pas des Facebook et twitoos qui n'ont jamais vu un stade.

Les attaques peuvent être personnalisées, mais il y a aussi celles qui touchent un groupe de personnes (par exemple les supporters d'un autre club). Dans le cas de (34), nous avons affaire à un rageux qui cherche à provoquer les supporters du Paris Saint-Germain en manifestant sa joie de l'élimination du club adverse en Coupe d'Europe.

(34) lorenlandes. apres l elimination de Paris c 'est une semaine tout simplement parfaite!

Il arrive que de tels propos soient l'objet de réactions. Dans l'exemple du même type (35) qui concerne une situation similaire d'élimination d'un club populaire (ici le F.C. Barcelone), il ne faut pas attendre longtemps pour les réactions des supporters du club catalan qui ripostent (36) :

(35) Melo229. ma journée devient bien meilleure.

(36) steven_zidane. Ça va, pas trop fade ta vie? Relie ton com stp et cite le à haute voie, tu te sentiras seul...

ou

(37) rateau. Ta vie doit être bien triste mon pauvre.

L'exemple (35) ainsi que les réactions (36) et (37) illustrent bien comment agissent les rageux qui savent comment provoquer les autres et accomplir leur désir 
de nuire à autrui. Dans les propos que tiennent les internautes et qui est un des traits caractéristiques du hate, on retrouve des insultes. L'insulte que nous analysons ici comme acte de parole cherchant à outrager ou constituant un outrage est utilisée dans des buts de provocation, de manipulation ou tout simplement comme échappatoire pour calmer la frustration. Les insultes sont en général personnalisées comme les exemples sous l'article II :

(38) Luigimatula. Je n'ai jamais aimé Drogba. Pourtant fan de l'om. Je ne comprends pas qu'on aime les grosses têtes comme lui.

(39) MohaBitaNegra. Espèce de voleur.

L'utilisation des formes grosses têtes et voleur sont le résultat du contenu de l'article qui a incité à cette réaction (39) ou qui a servi de support pour manifester son opinion défavorable envers un protagoniste non apprécié. Ceci est aussi le cas de exemples qui suivent :

(40) fouteux28. lui, il se serait excusé de ce qu'a fait benz et aurait lu la lettre de remords de benz a valb. PITRE va.

(41) Pierre.R06. Il est totalement disqualifié pour émettre un avis. Comment peut on encore tendre un micro à ce parangon.

Les insultes PITRE et parangon sont ici des avis personnels des auteurs des commentaires qui n'ont pas pu résister à la tentation d'ajouter leur grain de sel à la discussion. Une méthode fréquemment utilisée par les haters c'est l'utilisation de l'hypercorrection afin de rabaisser les propos des autres internautes. La communication sur internet se fait d'une façon instantanée et la forme du message ne devrait pas être jugée. Les règles d'orthographe et de grammaire sont en général profanées, car le but principal est de transmettre une idée à travers un message dont c'est le sens qui sera l'objet du jugement des autres participants de l'interaction. Toutefois, il arrive parfois que faute d'idées ou par volonté de manifester son refus envers certains propos, il y a des internautes qui signalent les manques de connaissances dans le domaine du bon français. Cela aboutit parfois à des situations insolites comme dans les exemples d'échanges qui suivent.

(42) bichon83. pour 1 euros ok.

(43) JL11301. Tu dois pas savoir compter toi pour mettre 1 euro au pluriel? Allez bisous de moi!

(44) recreativohuelva. Ou un s de trop plutôt.

Dans cet échange on retrouve un commentaire persifleur (43) qui n'a pas été apprécié par un adversaire qui a considéré qu'il fallait punir l'opposant en lui signalant une faute dans son énoncé (43). Cependant la riposte fut manquée du fait qu'un observateur de l'échange a remarqué une incohérence dans cette réponse et il n'a pas 
hésité à signaler l'erreur (44). Ce type d'échanges où les internautes interviennent en fonction de leur support ou refus pour l'idée de l'intervenant n'est pas rare, en voici encore un exemple.

(45) ghot. Logique normal il n'a jamais aimer la France !!!!!!!!!!!

(46) le_sochalien_25600. Et tu n'aimes pas l'orthographe apparemment...

(47) le_glaude. Et toi, commence par aimer le français ...

(48) ascannes007. Toi aussi en premier car c'est "commenceS", merci, au revoir :).

Dans ce dialogue entre internautes, il faut remarquer qu'en général ce sont les tenants de différents points de vue qui s'affrontent dans de tels échanges. Dans le cas des exemples (45), (46), (47), (48) il s'agit des supporters et des adversaires du footballeur Karime Bezema qui fait naître beaucoup d'émotions chez les internautes du fait de son comportement immoral (L'Affaire Zahia ${ }^{17}$ ) et antipatriotique (obstination à ne pas chanter l'hymne national).

\section{CONCLUSION}

Le discours qui se tient sur internet est le résultat de l'apparition de ce moyen de communication qui a donné naissance à un nouveau type de polémique. Les commentaires sur internet sont accessibles à tous, cependant il existe certaines règles et coutumes qui font des participants de ce terrain d'échanges verbaux des membres d'une communauté qui est soumise à des règles et des codes qui relèvent d'un accord tacite. Parmi les commentaires qui sont "postés » sur la toile, nombreux sont ceux qui ressortent d'un phénomène récent qui constitue une particularité de l'internet - le hate. Le hate diffère du hate speech et du trolling quoique parfois la limite semble être très floue. Les internautes qui s'adonnent à ce type de pratiques sont connus sous une appellation générale - les rageux (haters). Le but de ces personnes est surtout de provoquer, persifler et ridiculiser tant le contenu des articles de presse que les propos écrits par d'autres participants des échanges verbaux sur la toile. Se pencher vers le hate est une attitude volontaire qui résulte d'un côté des traits de la personnalité (manque de confiance en soi, besoin d'évacuer la colère ou de se mettre en valeur) et d'une tendance globale qui considère ce type de pratiques comme un jeu malicieux auquel participe un grand nombre d'internautes. Les commentateurs du football sur internet forment une micro-societé, ils possèdent leur propre modèle de comportements qui établit des règles qui déterminent ce qui est apprécié ou pas. Les autres internautes jouent le rôle de juges dans ce jeu qui peut être blessant pour ceux qui n'en comprennent pas les règles. Le hate constitue un

${ }^{17}$ Affaire du monde de football où deux joueurs de l'Équipe de France de football ont été inculpés pour avoir utilisé les services d'une prostituée mineure. 
trait propre de la langue des rageux, il a une fonction identitaire pour ces gens souvent habitués et dépendants des forums et des réseaux sociaux qui constituent pour eux un lieu de deuxième vie dans laquelle ils peuvent se défouler pour compenser les échecs de la vie réelle. Le hate est un type de discours très riche en créations lexicales, figures et arguments qui peuvent être analysés à l'aide des outils que fournit l'analyse du discours.

\section{INDEX DES COMMENTAIRES}

I. Blanc a perdu gros (du 14/04/2016)

(3) Orvis. "Ahhh, vous voyez bien que je sais prendre les bonnes décisions!". Au secours

(7) JMHoleAss. tu vends des joueurs en fin de contrat? je te prends comme conseiller patrimonial si t'es libre.

(21) philou-21. blanc n 'a rien perdu puisque blanc n 'a rien...gagné. et ce psg kakari ne gagnera JAMAIS rien. (kakari pour qatari)

(27) adyabeba. Le PSG ne gagnera pas la LDC... sans entraineur...

(28) Garcia. En 1995, le PSG était allé en demi-finale contre AC Milan (...) Ils existaient donc plus que le grand joueur de ligue 1 mais petit joueur de Cl qu'est MONSIEUR Ibrahimovic.

(29) JMHoleAss. Peut être mais la Prince a publiquement applaudi le retour du cousin.

II. La fondation de Didier Drogba accusée de fraude (du 14/04/2016)

(8) vivemezigue. J'ai décidé de créer un fondation en ma faveur avec un but lucratif, je vous remercie de votre générosité.

(14) Giman. Un hôpital vide, c est plutôt une bonne nouvelle ...

(20) THE_CRITICAL_ONE. Un Ivoirien accusé de Fraude, il va encore nous dire qu'il n'a rien vu.

(24) f_philippot. Les africains sont coutumiers du fait, souvenez-vous Boli (tiens, un ancien marseillais aussi) et mouss diouf (pour ne citer qu'eux) et après ça vient donner des leçons aux autres.

(25) waldemar victorino. étonnant, de la part d'un ancien soutien de Gbagbo, vraiment étonnant...

(38) Luigimatula. Je n'ai jamais aimé Drogba. Pourtant fan de l'om. Je ne comprends pas qu'on aime les grosses têtes comme lui.

(39) MohaBitaNegra. Espèce de voleur.

III. La vente de l'OM, "une bonne nouvelle » selon Luc Laboz, le directeur général du club (du 14/04/2016)

(5) vivemezigue. Recherche repreneur inconscient capable de mettre beaucoup d'argent, sans retour sur investissement, pour remettre en état un club en ruines non entretenu, avec des supporters insupportables des joueurs sans volonté. Prévoir dirigeants compétents et directeur sportif connaissant le football ainsi qu'un entraineur avec le même profil. Faire offre. 
(9) MyKeulKeul69. Le tout est de retrouver un repreneur sérieux. De toute façon, la situation actuelle de l'OM ne doit pas durer. Parce que là, on rigole, mais depuis la dernière victoire Marseillaise au Vélodrome, on a changé 2 fois d'heure :-)

(10) Yeshol. Kobe va racheter l'OM.

(11) abas64. Gilbert montagné est sur le coup.

(12) Dearduke. Il ne reste plus qu'à faire venir Stéphane Plaza pour faire du club staging, et trouver un acheteur:)

(13) StellaRossa. Que la fête commence! C'est la tournée des charlots aux pays des sardines. 1, 2, 3 dis Kachkar!

(15) Le coq Gaulois. En espérant un repreneur aussi propre que les Qataris à Paris, juste pour voir comme ça les réactions de nos chers amis Marseillais...

(19) pascaloudu13. les vautours à l'affut se positionnent (DIOUF ANIGO).

(26) THE_CRITICAL_ONE. Ya bien des pigeons qui ont rachetés le PSG, alors un vrai club avec une vraie histoire et des vrais supporters ça va se vendre facilement.

(31) syl2alf. On reconnait bien les lâches, passant de la flagornerie au lynchage public! Ils sont beaux les corbeaux de l'Equipe! Mais qui voudrait payé pour lire de pareils sornettes, cachez vous derrière le payant, vos étrons n'en seront que moins nocifs...

(32) Hubert-ALLESSE. syl +50 .

(33) nasri_13300. stella l'élu des Charlot c'est bien toi juste pour info l om est toujours le club le plus titré et le plus suivit de France . je parle pas des Facebook et twitoos qui n'ont jamais vu un stade.

(42) bichon83. pour 1 euros ok.

(43) JL11301. Tu dois pas savoir compter toi pour mettre 1 euro au pluriel ?Allez bisous de moi!

(44) recreativohuelva. Ou un s de trop plutôt.

IV. Karim Benzema : Raymond Domenech aurait pris la même décision que la FFF (du 14/04/2016)

(1) greg_44. C'est qui lui? On s'en bat les...

(2) arctarus. Qu'en pense Aimé Jacquet ? Et même Michel Hidalgo ? et ma concierge.

(4) LeParisienDu12. Quelle pipelette, la Raymonde!

(16) cariocabrazilian. Qui s'en fout ????

(17) Padawan44. Tu le sors par la porte, il revient par la fenetre, tactiquement trop fort Raymond, comme tes tacles par derrière.

(18) lamolie. ce mec est pire qu'un virus qu'il se taise à jamais.

(41) Pierre.R06. Il est totalement disqualifié pour émettre un avis. Comment peut on encore tendre un micro à ce parangon

V. Bleus : Karim Benzema ne sera pas sélectionné pour l'Euro (du 13/04/2016)

(6) phil15. t'as pas les numéros sortants du prochain loto, stp?

(22) gil06. Super bêtezema aura du temps pour apprendre la marseillaise.

(30) groove33. Ouais allee parlons du fc pepito et du coupeur de citrons d'arsenal.

(40) fouteux28. lui, il se serait excusé de ce qu'a fait benz et aurait lu la lettre de remords de benz a valb. PITRE va

(45) ghot. Logique normal il n'a jamais aimer la France !!!!!!!!!!! 
(46) le_sochalien_25600. Et tu n'aimes pas l'orthographe apparemment...

(47) le_glaude. Et toi, commence par aimer le français...

(48) ascannes007. Toi aussi en premier car c'est “commenceS”, merci, au revoir :)

VI. Atlético-Barça : la main de Gabi était-elle dans la surface? (du 13/04/2016)

(23) fdcap. eh oh les cakalents elle a joué la messi car pas vu.

(34) lorenlandes. apres $l$ elimination de Paris $c$ 'est une semaine tout simplement parfaite!

(35) Melo229. ma journée devient bien meilleure.

(36) steven_zidane. Ça va, pas trop fade ta vie? Relie ton com stp et cite le à haute voie, tu te sentiras seul...

(37) rateau. Ta vie doit être bien triste mon pauvre. 


\section{BIBLIOGRAPHIE}

Ferrier, B. (2013). GRODICO, le dictionnaire du français tel qu'on le parle. Paris : Éditions de l'œuvre. Labov, W. (1993). Le parler ordinaire. Paris : Les éditions de Minuit.

Reboul, O. (2011). Introduction à la rhétorique. Paris : QUADRIGE/Presses Universitaires de France.

Sablayrolles, J.-F. (2015). Quelques remarques sur une typologie des néologismes: Amalgamation ou téléscopage : un processus aux productions variées (mots valises, détournements) et un tableau hiérarchisé des matrices. In I. M. Alves et E. S. Pereira (éds), Neologia das linguas romanicas. São Paulo : Humanitas, pp. 187-218.

Sablayrolles, J.-F. (2017). Les néologismes. Créer des mots français aujourd'hui. Paris: Éditions Garnier. 